\title{
Osteoporosis and the Role of Physical Therapy in the Different Domains
}

Gabriella Posa, Erika Roka, Edit Sziver, Regina Finta, Levente Szilágyi, Krisztina Koncsek PT and Edit Nagy*

Faculty of Health and Social Sciences, Department of Physiotherapy, University of Szeged, Hungary

"Corresponding author: Edit Nagy, University of Szeged, Faculty of Health and Social Sciences, Department of Physiotherapy, Temesvari krt 31. Szeged, Hungary, Tel: +36 62 545037; E-mail: nedit@etszk.u-szeged.hu

Received date: January 20, 2017; Accepted date: February 09, 2017; Published date: February 16, 2017

Copyright: (C) 2017 Posa G, et al. This is an open-access article distributed under the terms of the Creative Commons Attribution License, which permits unrestricted use, distribution, and reproduction in any medium, provided the original author and source are credited.

\begin{abstract}
The authors emphasize the role of physical exercises in the prevention and treatment of osteoporosis through reviewing the most important evidences. With the increase of life expectancy, it becomes more and more challenging to fight against osteoporosis related fracture complications and functional restrictions. The article gives an insight into the different physical exercises that are able to influence the condition as prevention and also as treatment possibilities without having adverse side effects. Since improving balance and postural control are essential part of the interventions and they are under the influence of multiple sensory, motor and cognitive systems, this multisystem nature of balance should take into account in falls prevention even in terms of osteoporosis.
\end{abstract}

Keywords: Osteoporosis; Bone loss; Balance; Fall prevention; Exercise

Abbreviations OP: Osteoporosis; SD: Standard deviation; WHO: World Health Organisation; BMD: Bone mineral density; BBS: Berg Balance Scale; ML: Mediolateral; AP: Anteroposterior; SPPB: Short Physical Performance Battery

\section{Introduction}

\section{The scope of the problem}

Osteoporosis (OP) is characterized by reduced bone mass and disruption of bone architecture, resulting in increased risk of fragility fractures which represent the main clinical consequence of the disease. Fragility fractures are associated with substantial pain and suffering, disability and even death for affected patients and substantial costs to society [1].

OP is a global health problem all around the world. The number of osteoporotic fractures is rising in many countries. Reasons for this relate in part to the increased longevity of the population.

The prevalence of osteoporosis in the EU is estimated at 27.6 million in 2010, out of this 5, 6 million were male [1]. Improvements in socioeconomic prosperity that in turn decrease everyday levels of physical activity may be a factor associated with increasing fracture rates. In Europe, the total population will not increase markedly over the next 25 years, but the proportion accounted for by the elderly will increase by $56 \%$ in men and by $41 \%$ in women [2].

\section{Bone loss}

One of the important predictors of the future fractures is the bone mineral density (BMD). Bone mineral density is most often described as a T- or Z-score, both of which are units of standard deviation (SD). The T-score describes the number of SDs by which the BMD in an individual differs from the mean value expected in young healthy individuals. Each standard deviation decrease in bone density is associated with a twofold increase in fracture risk [3]. The operational definition of osteoporosis is based on the T-score for BMD assessed at the femoral neck and is defined as a value for BMD 2.5 SD or more below the young female adult mean $[4,5]$.

Immobilisation is an important cause of bone loss. Immobilised patients may lose as much bone in a week when confined to bed than they would otherwise lose in a year. For this reason, immobility should, wherever possible, be avoided. The amount of weight-bearing exercise that is optimal for skeletal health in patients with osteoporosis is not known, but exercise forms an integral component of management at each level of prevention and treatment of osteoporosis [6].

\section{The different levels of prevention}

Primary prevention of the osteoporosis is aimed to ensure that women reach their maximal peak bone mass and minimise the bone loss during their early adult years. The peak bone mass is mainly controlled by genetic factors, but is influenced by lifestyle factors including adequate dietary calcium intake and physical exercise, as well as avoiding bone toxins like smoking and excess alcohol [7]. Cessation of tobacco use and avoidance of excessive alcohol intake is essential in prevention. Since the use of tobacco products is detrimental to the skeleton as well as to overall health $[8,9]$.

Secondary prevention focuses on the prevention of fractures in women who have osteopenia or osteoporosis according to the bone density measurements. This secondary prevention approach targets usually the peri- and postmenopausal women and those who are at high risk for secondary osteoporosis.

The tertiary prevention is the strategy to prevent future fractures in women with osteoporosis who have already sustained a fracture. The secondary and tertiary prevention includes lifestyle modification, pharmacologic and fall prevention strategies [10].

\section{Evidences about the effect of physical exercises on bone mass}

There are several evidences that regular weight-bearing and musclestrengthening exercises are recommended to improve agility, strength, 
posture, and balance; maintain or improve bone strength; and reduce the risk of falls and fractures [11]. These types of exercises helps to reduce the risk of falls and fractures in addition, exercise may modestly increase bone density [12-14].

Over the past decades several studies proved the positive effect of physical exercises on the bone mass. In the elderly, physical exercise is meant to prevent and to fight against the age-related and the postmenopausal bone loss. Nine postmenopausal women exercising for one hour three times a week (warm-up, conditioning and circulatory exercises) presented after one year an increase in the total body calcium (measured by total body neutron activation analysis) while a decrease was noted in a group of 9 sedentary women [15].

Thirty-six postmenopausal women aged 50-63 years, practising aerobic dancing with increasing frequency and intensity during 6 months, preserved their mineral bone content (measured by photon absorptiometry of the distal radius). Statistically significant amounts were lost during the same period in a control and in a walking group. Plasma oestrogen levels seemed to be not influenced by exercise, supporting the hypothesis that the main factor in prevention of the bone mass loss is the mechanical loading due to the physical exercises [16].

A program of dynamic loading exercises of the distal forearm (tension, torsion, compression, bending) applied three times a week for 5 months in 14 postmenopausal women aged 53-74 years showed a $3.8 \%$ increase in the mean bone density (measured by Compton scattering technique) and a decrease of $1.9 \%$ in the control group [17]. Investigating the effect of Pilates exercises on BMD enrolling 41 postmenopausal women, Angin et al. found that BMD values increased significantly in the Pilates group while the BMD decreased in the control group [18].

The effect of selected yoga postures were evaluated in case of 741 Internet-recruited volunteers comparing preyoga BMD changes with postyoga BMD changes. Bone mineral density improved in spine, hips, and femur of the 227 moderately and fully compliant patients, so it was suggested that selected yoga postures can raise BMD [19].

McArthur et al. emphasize the careful selection and adaptations of yoga postures to the osteoporotic patients in order to avoid the risk of injuries. Contraindicated movements include end-range flexion/ extension/rotation of the spine and internal/external rotation of the hip. Yoga postures that should be encouraged include postures emphasizing spinal alignment and extension to mid-range in standing and on the floor [20].

Zhao et al. synthesized current evidences from 24 clinical trials to evaluate the impact of different resistance training modes on postmenopausal bone loss. According to their meta-analysis the exercise interventions were categorized into two training modes, namely resistance-alone versus combined resistance training protocols. The combined resistance training protocols were defined as the combination of resistance training and high-impact or weight-bearing exercise. The results suggested that the combined resistance training protocols were effective in improving bone mineral density at the femoral neck and lumbar spine [21].

Although tai chi exercises became increasingly popular in the treatment of osteoporotic patients, according to the results of a recent meta-analysis, only limited conclusions can be drawn regarding the efficacy of tai chi exercise on bone health [22].
The negative effect of vigorous exercise and a concern have been also published, that many women athletes whose rigorous training schedules and restrictive dietary practices have led to extended periods of amenorrhea may have also suffered irreversible bone loss [23].

\section{Evidences about balance, postural control and functional exercises in fall prevention}

Since the prevalence osteoporosis increases with age, there is an overlap between falls prevention and balance training in case of elderly and osteoporotic patients.

According to Williams et al., elderly subjects exhibited significantly greater frequency dispersion than young adults in the ML direction. Moreover the elderly were characterized by a greater concentration of power at low frequencies in the ML postural forces. The appearance of a low-frequency component exhibited by the elderly suggests the emergence of a slow postural drift or a slow shifting of weight during static standing [24]. Over-reliance on hip joint action has been shown in the elderly when they respond to unexpected postural disturbances [25].

It is well known that older people with balance disorders suffer from multiple impairments, such as multi-sensory loss, weakness, orthopaedic constraints and cognitive impairments [26,27]. The decreased muscle strength is related to diminished postural control $[28,29]$ whereas, impaired postural control is associated with an increased risk of falling $[30,31]$ and elderly fallers have been reported to display a significant decrease in the dynamic strength of the muscles of the knees and ankles as compared with non-fallers [32]. Sziver et al. in a recent study assessing the postural control in patients with osteoarthritis (OA) and rheumatoid arthritis (RA) affecting the hip joint found, that in case of both hip joint impaired groups there was a significantly larger postural sway in comparison with the control group [33].

There are several studies providing evidences about the efficacy of balance training in case of elderly as a possible way in fall prevention. These results indicated that the improvement occurs as a decreased centre of force displacement, i.e., a better balance control means a smaller postural sway [32,34]. However, in our earlier study, we found that the balance training performed by elderly caused a significant improvement in functional performance, but a significantly longer sway path was observed after training in the ML direction.

The results suggest that the participants' balance confidence and the control of ML balance improved in response to the training. The higher ML frequency power exhibited after the training may be indicative of a better balance performance. Thus, the increase in the sway path in this special age group did not mean a further impairment of the postural control rather a better confidence to allow and being able to control higher sway amplitudes [35].

\section{Impairments related to aging and osteoporosis}

Among women, it has been shown that osteoporotic women have significantly lower back extensor strength than healthy women [36]. Moreover, besides the low back extensor weakness, there is weakness in the lower extremity muscle strength as well [37]. Significantly lower hip abductor strength was found in osteoporotic subjects with kyphosis, which resulted in greater ML displacement of the centre of mass [38] and it is well known, that the increased ML postural sway is the predictor of falls [39]. 
The increased kyphosis has been associated with other medical problems, such as falls [40]. It is interesting to note that in the study of Eum and co-workers, the kyphosis was not associated with balance performance as measured by the Berg Balance Scale (BBS) or within the balance component of the Short Physical Performance Battery (SPPB) [41]. In contrary to this, Antonelli-Incalzi and colleagues found that increased kyphosis was associated with impaired balance in women, where balance was measured using a testing procedure that is similar to the balance component of the SPPB [42]. Greig et al. found involving 22 osteoporotic women to their study, vertebral fracture to be related to impaired balance characteristics, rather than thoracic kyphosis [43]. This might suggest that a history of fracture is a factor that is influencing balance rather than kyphosis.

\section{Dual task situations, cognitive processing as a risk in falls}

Decrements in dual-task postural control performance (simultaneously performing two tasks, at least one of which requires postural control) have been associated with an increased risk of falling. Among older adults, impairment in the control of balance under dualtask conditions is a common occurrence therefore interventions that improve dual-task balance performance are a critical health care need $[44,45]$.

A randomized controlled trial provides evidence that an individualized training program was effective in improving balance under single-task contexts in older adults with balance impairment. After the 4-week intervention program, participants in all training groups significantly improved performance on single-task gait speed and the BBS. Even though both single-task and dual-task training programs were equally effective at improving balance and walking performance under single-task conditions, dual-task training programs were superior to single-task training in improving walking under dualtask contexts in case of the elderly subjects with balance impairments [46].

Konak et al. found that a 4-week single- and dual-task balance exercise programs were effective in improving static balance, dynamic balance and balance confidence during daily activities in older adults with osteoporosis. However, single- and dual-task gait speeds showed greater improvement following the application of a specific type of dual-task exercise programs [47].

\section{Discussion and Conclusion}

There is a bulk of evidence about the effectively of physical exercises both on the bone health and on balance performance and there is an agreement about the general benefits of physical exercises in prevention of osteoporosis. As well as the negative effect of vigorous training and the restrictive dietary practices in elite women athletes has been emphasized.

Since balance and postural control are under the influence of multiple sensory, motor and cognitive systems, this multisystem nature of balance should take into account in falls prevention even in terms of osteoporosis when assessing the risk of falling and planning interventions through physical exercises to prevent it. Because each individual has unique set of system constraints and resources available to control posture and balance, the ability to maintain equilibrium and postural orientation will depend on the particular context. Thus, different persons will fall in different situations, depending on what systems are required to complete the task successfully [48]. As Horak suggested, in order to predict risks of falling and to design an optimal intervention for persons with balance impairments, it is important to assess the integrity of underlying physiological systems (sensory, motor and cognitive) and compensatory strategies available. Therefore simple global measures of 'balance' are insufficient to provide the information for prediction of the falls [48].

\section{Conflict of Interest}

The authors declare that they have no competing interests.

\section{References}

1. Hernlund E, Svedbom A, Ivergård M, Compston J, Cooper C, et al. (2013) Osteoporosis in the European Union: medical management, epidemiology and economic burden a report prepared in collaboration with the International Osteoporosis Foundation (IOF) and the European Federation of Pharmaceutical Industry Associations (EFPIA). Arch Osteoporos 8: 136.

2. http://www.un.org/en/development/desa/population/

3. Ullom-Minnich P (1999) Prevention of osteoporosis and fractures. Am Fam Physician 60: 194-202.

4. World Health Organisation (1994) Assessment of fracture risk and its application to screening for postmenopausal osteoporosis. Report of a WHO Study Group. World Health Organ Tech Rep Ser 843: 1-129.

5. Kanis JA, Melton LJ, Christiansen C, Johnston CC, Khaltaev N (1994) The diagnosis of osteoporosis. J Bone Miner Res 9: 1137-1141.

6. Kanis JA, McCloskey EV, Johansson H, Cooper C, Rizzoli R, et al. (2013) European guidance for the diagnosis and management of osteoporosis in postmenopausal women. Osteoporos Int 24: 23-57.

7. Soyka LA, Fairfield WP, Klibanski A (2000) Clinical review 117: Hormonal determinants and disorders of peak bone mass in children. J Clin Endocrinol Metab 85: 3951-3963.

8. Krall EA, Dawson-Hughes B (1999) Smoking increases bone loss and decreases intestinal calcium absorption. J Bone Miner Res 14: 215-220.

9. Maurel DB, Boisseau N, Benhamou CL, Jaffre C (2012) Alcohol and bone: review of dose effects and mechanisms. Osteoporos Int 23: 1-16.

10. Schrager S (2003) Osteoporosis prevention in primary care. WMJ 102: 52-55.

11. Cosman F, de Beur SJ, LeBoff MS, Lewiecki EM, Tanner B, et al. (2014) Clinician's guide to prevention and treatment of osteoporosis. Osteoporos Int 25: 2359-2381.

12. Granacher U, Gollhofer A, Hortobágyi T, Kressig RW, Muehlbauer T (2013) The importance of trunk muscle strength for balance, functional performance and fall prevention in seniors: A systematic review. Sports Med 43: 627-641.

13. Sherrington C, Whitney JC, Lord SR, Herbert RD, Cumming RG, et al. (2008) Effective exercise for the prevention of falls: A systematic review and meta-analysis. J Am Geriatr Soc 56: 2234-2243.

14. Choi M, Hector M (2012) Effectiveness of intervention programs in preventing falls: A systematic review of recent 10 years and meta-analysis. J Am Med Dir Assoc 13: 188.

15. Aloia JF, Cohn SH, Ostuni JA, Cane R, Ellis K (1978) Prevention of involutional bone loss by exercise. Ann Intern Med 89: 356-358.

16. White MK, Martin RB, Yeater RA, Butcher RL, Radin EL (1984) The effects of exercise on the bones of postmenopausal women. Int Orthop 7: 209-214.

17. Simkin A, Ayalon J, Leichter J (1987) Increased trabecular bone density due to bone-loading exercises in postmenopausal osteoporotic women. Calcif Tissue Int 40: 59-63.

18. Angin E, Erden Z, Can F (2015) The effects of clinical pilates exercises on bone mineral density, physical performance and quality of life of women with postmenopausal osteoporosis. J Back Musculoskelet Rehabil 28: 849-58. 
19. Lu YH, Rosner B, Chang G, Fishman LM (2016) Twelve-minute daily yoga regimen reverses osteoporotic bone loss. Top Geriatr Rehabil 32: 81-87.

20. McArthur C, Laprade J, Giangregorio LM (2016) Suggestions for adapting yoga to the needs of older adults with osteoporosis. J Altern Complement Med 22: 223-226.

21. Zhao $\mathrm{R}, \mathrm{Zhao} \mathrm{M}, \mathrm{Xu} \mathrm{Z}$ (2015) The effects of differing resistance training modes on the preservation of bone mineral density in postmenopausal women: A meta-analysis. Osteoporos Int 26: 1605-1618.

22. Sun Z, Chen H, Berger MR, Zhang L, Guo H, et al. (2016) Effects of tai chi exercise on bone health in perimenopausal and postmenopausal women: A systematic review and meta-analysis. Osteoporos Int 27: 2901-2011.

23. Keen AD, Drinkwater BL (1997) Irreversible bone loss in former amenorrheic athletes. Osteoporos Int 7: 311-315.

24. Williams HG, McClenaghan BA, Dickerson J (1997) Spectral characteristics of postural control in elderly individuals. Arch Phys Med Rehabil 78: 737-744.

25. Manchester D, Wollacott M, Zederbauer-hylton N, Marin O (1989) Visual, vestibular and somatosensory contributions to balance control in older adult. J Gerontol Med Sci 44: M118-M127.

26. Lord SR, Ward JA, Williams P, Anstey KJ (1994) Physiological factors associated with falls in older community-dwelling women. J Am Geriatr Soc 42: 1110-1117.

27. Tinetti ME, Inouye SK, Gill TM, Doucette JT. (1995) Shared risk factors for falls, incontinence and functional dependence: Unifying the approach to geriatric syndromes. J Am Med Assoc 273: 1348-1353.

28. Wolfson L, Judge J, Whipple R, King M (1995) Strength is a major factor in balance, gait, and the occurrence of falls. J Gerontol A Biol Sci Med Sci 50: 64-67.

29. Onambele GL, Narici MV, Maganaris CN (2006) Calf muscle-tendon properties and postural balance in old age. J Appl Physiol (1985) 100 2048-2056.

30. Campbell AJ, Borrie MJ, Spears GF (1989) Risk factors for falls in a community-based prospective study of people 70 years and older. Gerontol 44: M112-117.

31. Lord SR, Ward JA, Williams P, Anstey KJ (1994) Physiological factors associated with falls in older community-dwelling women. J Am Geriatr Soc 42: 1110-1117.

32. Whipple RH, Wolfson LI, Amerman PM (1987) The relationship of knee and ankle weakness to falls in nursing home residents: An isokinetic study. J Am Geriatr Soc 35: 13-20.

33. Sziver E, Nagy E, Preszner-Domján A, Pósa G, Horvath G, et al. (2016) Postural control in degenerative diseases of the hip joint. Clin Biomech (Bristol, Avon) 35: 1-6.

34. Hue OA, Seynnes O, Ledrole D, Colson SS, Bernard PL (2004) Effects of a physical activity program on postural stability in older people. Aging Clin Exp Res 16: 356-362.
35. Nagy E, Feher-Kiss A, Barnai M, Domján-Preszner A, Angyan L, et al. (2007) Postural control in elderly subjects participating in balance training. Eur J Appl Physiol 100: 97-104.

36. Hongo M, Itoi E, Sinaki M, Miyakoshi N, Shimada Y, et al. (2007) Effect of low-intensity back exercise on quality of life and back extensor strength in patients with osteoporosis: A randomized controlled trial. Osteoporos Int 18: 1389-1395.

37. Sinaki M, Khosla S, Limburg PJ, Rogers JW, Murtaugh PA (1993) Muscle strength in osteoporotic versus normal women. Osteoporos Int 3: 8-12.

38. Sinaki M, Brey RH, Hughes CA, Larson DR, Kaufman KR (2005) Balance disorder and increased risk of falls in osteoporosis and kyphosis: significance of kyphotic posture and muscle strength. Osteoporos Int 16: 1004-1010.

39. Maki BE, Holliday PJ, Topper AK (1994) A prospective study of postural balance and risk of falling in an ambulatory and independent elderly population. J Gerontol 49: M72-84.

40. Kasukawa Y, Miyakoshi N, Hongo M, Ishikawa Y, Noguchi H, et al. (2010) Relationships between falls, spinal curvature, spinal mobility and back extensor strength in elderly people. J Bone Miner Metab 28: 82-87.

41. Eum R, Leveille SG, Kiely DK, Kiel DP, Samelson EJ, et al. (2013) Is kyphosis related to mobility, balance and disability? Am J Phys Med Rehabil 92: 980-989.

42. Antonelli-Incalzi R, Pedone C, Cesari M, Di Iorio A, Bandinelli A, et al. (2007) Relationship between the occiput-wall distance and physical performance in the elderly: A cross sectional study. Aging Clin Exp Res 19: 207-212.

43. Greig AM, Bennell KL, Briggs AM, Wark JD, Hodges PW (2007) Balance impairment is related to vertebral fracture rather than thoracic kyphosis in individuals with osteoporosis. Osteoporos Int Apr 18: 543-551.

44. Beauchet O, Dubost V, Herrmann F, Rabilloud M, Gonthier R, et al. (2005) Relationship between dual-task related gait changes and intrinsic risk factors for falls among transitional frail older adults. Aging Clin Exp Res 17: 270-275.

45. Hausdorff JM, Yogev G, Springer S, Simon ES, Giladi N (2005) Walking is more like catching than tapping: Gait in the elderly as a complex cognitive task. Exp Brain Res 164: 541-548.

46. Silsupadol P, Shumway-Cook A, Lugade V, van Donkelaar P, Chou LS, et al. (2009) Effects of single-task versus dual-task training on balance performance in older adults: A double-blind, randomized controlled trial. Arch Phys Med Rehabil 90: 381-387.

47. Konak, HE, Kibar, S, Ergin, ES (2016) The effect of single-task and dualtask balance exercise programs on balance performance in adults with osteoporosis: A randomized controlled preliminary trial. Osteoporos Int 27: 3271-3278.

48. Horak FB (2006) Postural orientation and equilibrium: What do we need to know about neural control of balance to prevent falls? Age Ageing 35 Suppl 2: ii7-7ii11. 